\title{
Pensar e indagar la construcción social del espacio: balances y retos
}

\author{
Thinking and questioning the social construction \\ of space: balances and challenges
}

\author{
Nubia Moreno Lache ${ }^{1}$ \\ Alexander Cely Rodríguez ${ }^{2}$ \\ Liliana Rodríguez Pizzinato ${ }^{3}$
}

\section{Resumen}

Este artículo presenta un balance y reflexiones del trabajo realizado en la línea de investigación “Construcción social del espacio" 4 del programa de Maestría en Estudios Sociales en el Departamento de Ciencias Sociales de la Universidad Pedagógica Nacional. El propósito es investigar sobre la interrelación que la comunidad establece con el entorno biofísico y social, además del impacto cultural, económico y político -entre otros-en espacios geográficos, tales como la calle, el barrio, la localidad, la ciudad, la región e incluso el país. Asimismo, se trabajan categorías centrales asociadas a la identidad urbana, al espacio público, a la cognición ambiental del entorno urbano, a los comportamientos urbano-ambientales responsables, a la relación espacio y sujeto, y finalmente a la apropiación y significación de espacios públicos, territorios y territorialidades, producto de las interacciones humanas.

\section{Palabras clave:}

construcción social, investigación, espacio geográfico, espacialidad.

\section{Abstract}

This paper offers an overview and reflections of the research done within the emphasis on "Social Construction of Space", of the master's program in Social Sciences at the National Pedagogical University in Bogota, Colombia. The goal is to examine two issues: the relationships that community establishes with the biophysical and social environment and also the cultural, economical and political impact on geographic places, such as the street, neighborhood, town, city, region and even the country. Main categories such as urban identity, public space, environmental cognition of urban milieu, responsible urban and environmental behaviors and space/ subject relationships have been worked out. Finally, some studies have focused on the adoption and meaning of public spaces, territorialities and territories, as a result of human interactions.

Keywords:

social construction, research, geographical places, spatial category.

1 Universidad Distrital Francisco José de Caldas y Universidad Pedagógica Nacional, Bogotá, Colombia. Correo electrónico: nmorenol@pedagogica.edu.co

2 Universidad Pedagógica Nacional, Bogotá, Colombia. Correo electrónico: acely@pedagogica.edu.co

3 Universidad Distrital Francisco José de Caldas y Universidad Pedagógica Nacional, Bogotá, Colombia. Correo electrónico: Irodriguezp@pedagogica.edu.co

4 La línea de investigación es liderada por el grupo interinstitucional de investigación Geopaideia, lo que implica un trabajo en equipo que requiere la articulación de perspectivas y enfoques teóricos y metodológicos en el estudio de la espacialidad. Es relevante destacar los aportes, apoyo y recomendaciones brindados por la profesora Elsa Amanda Rodríguez de Moreno, gestora de la línea, así como del equipo docente de Geopaideia, quienes en eventos que se desarrollan al interior de la línea, en sesiones de socialización de proyectos, en salidas de campo y en acompañamiento de los trabajos de investigación, nutren y enriquecen esta experiencia. 


\section{La geografía: panorama de una disciplina fundamental para la compresión del espacio}

La topografía del valle es plana, pero ambos lados del río tienen partes altas, hacia las cordilleras que rodean el valle. El río es inmundo, color mierda, y los segundones soñamos con una corriente de agua que sea como el río Cristalino que hay en Tierra Fría. Los habitantes de Paradiso, en cambio, aburridos de tanto orden y control, (en su sector no hay ni siquiera un rastrojo) sueñan con pasar noches de farra en uno de los sektores de abajo; los habitantes de Boca del Infierno añoran un trabajo, una casa en $T$, un permiso para pasar temporadas en Paradiso. No quiero esquematizar ni dividir... la cosa es más ambigua de lo que parece, aunque obviamente en Paradiso, que ahora conozco mejor, viven los más ricos... No se sabe bien, en realidad, cuánta gente vive en Boca del Infierno;... la gente se esconde, no se deja entrevistar, temen ser expulsados aún más lejos, o que los datos sirvan como información para los operativos sangrientos de la Secur. Las cifras más precisas hablan de seis millones (pero podrían ser ocho si se cuentan las invasiones de desplazados hacia las lagunas fétidas de Babilonia...). En el mismo espacio, pero claro, con parques y avenidas y mejores servicios, vive medio millón de habitantes en Tierra Fría. Hay fábricas tanto arriba como abajo, pero son muy distintas y los obreros de las fábricas de Paradiso parecerían la élite limpia de los barrios menos astrosos del Sektor C, si pasaran a estos lados y los vieran.

(Abad, 2003, p. 140-141)

El saber geográfico ha recorrido varios caminos, encontrándose con diversas fronteras del conocimiento. Desde las propuestas originarias de la descripción, pasando por la representación del mundo, hasta la idea de la imagen que se tiene del mismo. Esta idea no estuvo alejada de las discusiones de lo que es ciencia y de lo que no lo es. A pesar de haber pasado casi dos siglos aún se oyen ecos de la vieja disputa entre el positivismo y otros paradigmas. Esto ha causado que la geografía, como ciencia y como saber, se debata entre las ciencias naturales y las ciencias sociales. De tal suerte, que es posible encontrarla ubicada en algunas universidades en las facultades de ciencias y en otras en las de humanidades. Es en Alemania que Humboldt y Ritter desarrollaron una visión de la geografía que estuvo más orientada hacia las ciencias naturales que a las ciencias sociales, y con la Revolución Industrial y el avance del imperialismo del siglo XIX se abrió el campo para el desarrollo de complejas técnicas de descripción, localización y medición.

En este contexto, el positivismo transfiere conceptos y teorías de las ciencias naturales (organismo, función, metabolismo) a las ciencias de la sociedad. Sobresalen las ideas de Darwin, Ratzel y Haeckel, que buscan el encadenamiento causal de los hechos y fomentan el desarrollo y el descubrimiento de nuevas leyes, hasta configurar el llamado determinismo geográfico. "Al determinismo geográfico le sucede el posibilismo según el cual el medio físico no determina las actividades, sino simplemente les ofrece posibilidades, que el hombre como ser libre, utiliza o desaprovecha" (Capel y Urteaga, 1985, p. 20). Otra visión corresponde al historicismo, que se preocupa por obtener una comprensión de la realidad sociocultural, vista en sus singularidades. Toma fuerza la geografía regional impulsada por Paul Vidal de la Blache, quien considera la región como área en la que se realizan las combinaciones particulares de fenómenos físicos y humanos caracterizados por un paisaje particular. Esta concepción ideográfica se aleja del carácter nomotético y no pretende encontrar leyes sino especificidades.

El siglo XX fue testigo de los vaivenes de la geografía como ciencia, desde el historicismo, que daría lugar al radicalismo, hasta el neopositivismo. En la década del treinta la geografía se orientaba a establecer un modelo, con base en complejas operaciones matemáticas, que diera razón de la localización y de la organización del entramado urbano aplicable a cualquier lugar. La teoría de la elección racional era su base más fuerte. Se adoptan conceptos provenientes de otras ciencias, se habla de física social, ecosistemas urbanos, entropía, gravitación y otros más para explicar el comportamiento espacial de las sociedades. 
La geografía humana, vista como una nueva manera de hacer y entender la geografía, busca según Ley y Samuels "reconciliar la ciencia social y el hombre, acomodar comprensión y juicio, objetividad y subjetividad, y materialismo e idealismo" (citados por Franco, 1997, p. 24). En otras palabras, considera al individuo como parte integrante fundamental de la comprensión en ciencias sociales, y aunque no se rechaza, ni mucho menos, la existencia de estructuras subyacentes, se plantea una ciencia social antropocéntrica en la que la acción y la conciencia humana desempeñen un papel activo y central. Otro importante representante de este enfoque y considerado como uno de sus pioneros es Yi-Fu Tuan con su trabajo "Humanistic Geography" (1976); su principal aporte radica en la contundente inmersión del sujeto como protagonista y actor central de los lugares, es decir que a partir de Tuan la geografía se dedica no solo a las esferas de lo biofísico sino que ingresa en el amplio y complejo escenario de la espacialidad como posibilidad de existencia, de habitar y de significación de los lugares.

En este escenario irrumpe la idea de que el espacio es organizado por las relaciones entre quienes lo habitan, esto significa que el individuo es un sujeto activo en relación con su entorno y acorde con su percepción; concepción denominada fenomenológica. A partir de 1960, el panorama geográfico encuentra otro punto paradigmático cuando se desarrolla la geografía de la percepción y las imágenes mentales. El objetivo fundamental es integrar este enfoque con otros campos del saber, específicamente con la psicología. Se parte de aceptar que el hombre es el principal elemento que modifica y complementa un espacio; por tanto lo esencial no es interpretar el medio geográfico real, sino identificar y analizar lo que percibe el hombre de acuerdo con sus vivencias y expectativas.

Ya que la geografía del comportamiento y de la percepción pretendía responder a las cuestiones planteadas en él: preocupación por el medio y los problemas sociales, orientación hacia la planificación y reconocimiento de los geógrafos, así como el resto de los mortales, son personas con
Nubia Moreno Lache, Alexander Cely Rodríguez, Liliana Rodríguez Pizzinato

visiones y percepciones diferentes del mundo, $y$ no observadores objetivos exentos de juicios de valor. (Estébanez, 1982, p. 93)

De manera simultánea, un sector importante de geógrafos aborda los conflictos sociales desde la perspectiva marxista, dando origen a la denominada geografía radical. Temas como la pobreza, el hambre, el desempleo, la marginación social, adquirieron un papel central. La búsqueda de sus causas y el compromiso con el cambio son sus banderas. Dicho enfoque es subsidiario de la teoría crítica de la Escuela de Frankfurt que influyó en "una nueva forma interdisciplinaria de estudiar los fenómenos geográficos sustentada en la epistemología materialista de Marx" (Franco, 1997, p. 21). Este enfoque plantea la necesidad de desarrollar un pensamiento geográfico social. Los geógrafos que han seguido el enfoque radical "han contribuido a la búsqueda del papel del geógrafo en el campo social desde una clara postura política y desde la teoría crítica de la sociedad". (Franco, 1997, p. 24)

La geografía radical, de acuerdo con Delgado (2003), define al espacio como producto social, históricamente constituido por la dinámica de los modos de producción, para ello los geógrafos llamados radicales acuden a los postulados fundamentales que estructuran el discurso marxista sobre el materialismo dialéctico-histórico. David Harvey (2001, citado por Delgado, 2003), analiza cómo las representaciones del espacio y del tiempo surgen de las prácticas sociales, pero son a su vez instrumentos de regulación de dichas prácticas. Por otra parte, Soja (1996, citado por Delgado, 2003) afirma que la espacialidad es el espacio socialmente construido por el conjunto de las relaciones intersubjetivas, económicas, políticas y culturales entre los individuos y los grupos. Siendo la espacialidad el resultado de la acción social sobre la naturaleza, como también de su propia dinámica social.

Delgado (2003) afirma que en la última década, surgen nuevos discursos que dan lugar a las denominadas geografías posmodernistas, desarrolladas a partir de la influencia de las filosofías posmodernas. Así, el nihilismo, la anarquía y demás tenden- 
cias individualistas entran en el discurso geográfico proponiendo acabar con los meta-relatos, con las tendencias emancipadoras calificadas de salvadoras o redentoras, por ejemplo geografías de género, geografía y ciberespacio, etnogeografías, geografías literarias, entre otras. (Delgado, 2003). Los discursos del posmodernismo se sustentan en la antilógica del poder, cuestionando los paradigmas tradicionales de la ciencia. Es decir, el campo de debate ya no es entre el espacio relativo y absoluto, sino entre lo micro y lo macro, entre lo local y lo mundial, entre la individualidad y la universalidad, entre lo homogéneo y lo heterogéneo, entre lo construido y producido históricamente y lo efímero, abstracto e irreal.

El panorama actual de la geografía permite reconocer tanto la presencia de estudios y tradiciones clásicas preocupadas por la explicación de los fenómenos e interacciones hombre-medio biofísico, como también el desarrollo de nuevos campos de saber geográfico. Estos se nominan como campos emergentes de la geografía, los cuales promueven y lideran nuevas y diversas maneras en la comprensión del espacio y por tanto abogan por la instauración de la espacialidad como categoría central en geografía. Interesa en estas perspectivas la relación de los sujetos con su entorno, la vivencia y experiencia espacial, el espacio como producto socioeconómico y sociocultural, los lugares específicos en donde se desarrolla la vida de las personas (casa, calle, parque, barrio, plaza, etc.); a su vez exponen estas perspectivas geográficas un fuerte interés por el desarrollo de una educación geográfica que considere los escenarios escolares como constructores de la espacialidad en los sujetos, pero que a su vez sea capaz de desbordarlos de modo que los ciudadanos habitantes de los lugares cimienten una relación de diálogo con el espacio que ocupan, alcanzando en lo posible la condición de habitar los lugares.

\section{La construcción social del espacio y su perspectiva de indagación}

La línea de investigación orienta su trabajo hacia la generación y realización de proyectos que puedan producir impacto en las comunidades y en las situa- ciones específicas en las cuales se desarrollan cada una de las indagaciones, aportando así elementos de estudio que nutran la problematización de la construcción social del espacio.

En armonía con ello, se pretende que los estudiantes de la línea aborden problemas contemporáneos demandantes de análisis y posibilidades de compresión y quizás solución en el espacio geográfico en general; se espera que a partir de estos hallazgos sea posible innovar en el campo profesional en el que se desempeñan, producir conocimiento, aportar soluciones a problemas teóricos y prácticos y presentar lineamientos que sirvan para diseñar políticas públicas afines con los asuntos de la espacialidad.

No obstante, los lineamientos considerados a partir del desarrollo de la experiencia investigativa con los estudiantes in situ, genera una serie de modificaciones en los aspectos teóricos y metodológicos en armonía con la realidad socio-espacial, y amplían el escenario de indagación de los asuntos territoriales, evidenciando lo multifacético y nutrido de estudiar la espacialidad.

La línea de investigación "Construcción social del espacio", tiene como objetivo fundamental ahondar en procesos de indagación en la interrelación comunidad-espacio geográfico, situación que se refleja en los desarrollos alcanzados y que muestran los siguientes horizontes conceptuales: estudio y comprensión de los espacios públicos, los territorios y las territorialidades urbanas, el sentido de lugar, las imágenes de ciudad, las prácticas docentes en la configuración de la espacialidad, los conceptos espaciales en textos escolares, la cartografía musical de la ciudad, la tenencia de la tierra, entre otros.

Este horizonte de indagación espacial se armoniza con el desarrollo de los campos emergentes de la geografía; si bien existe en la línea un fuerte componente del estudio de la geografía urbana y humana, los escenarios diversos que han emergido acuden y encuentran en estos campos fundamentos teóricos y metodológicos claros y precisos para abordar dichos estudios; en esta dirección es importante resaltar el auge en la línea de las denominadas geografías de 
la vida cotidiana, pues ellas hacen fuerte hincapié en la comprensión de la espacialidad a la luz de la simplicidad de la vida cotidiana de las personas, pero hay una enorme información que brinda el espacio habitado y usado cotidianamente por sus habitantes y que exige su estudio de manera rigurosa y científica; toda vez que estas geografías sostienen que en los espacios concretos e inmediatos los seres humanos desarrollan nutridas y complejas interacciones puesto que las

[...] prácticas espacio -temporales de los individuos y los hogares siguiendo trayectorias diarias, considerando sus movimientos, rupturas, los tiempos empleados y la secuencia de "estaciones", incluyendo el hogar, el trabajo, la iglesia, las compras, la escuela, el ocio, las actividades comunitarias [...] uno de los objetivos [...] es proporcionar un lenguaje sistemático y una metodología para describir el comportamiento espacial cotidiano, incluso para intervenir en las restricciones con las que se encuentran las personas [...] por eso va a diseñar una técnica que le permita representar tridimensionalmente las prácticas: los prismas espacio-temporales. (Lindón, 2006, p. 365).

\section{Senderos investigativos}

La relevancia del estudio del espacio social y sus diversas formas de conocimiento se estudian a través de diferentes estrategias didácticas que buscan complementar y complejizar su abordaje. Al respecto algunas de las actividades corresponden al desarrollo de seminarios teóricos y metodológicos de énfasis que aportan a la formulación, diseño metodológico y análisis de resultados de los proyectos, así como al proceso de escritura de las tesis.

Este proceso se complementa con la planeación y ejecución de conferencias nacionales e internacionales con expertos en temas específicos de los asuntos espaciales, que permiten contrastar otras formas de investigar el espacio geográfico; también resulta valiosa la participación como ponentes en congresos y eventos ${ }^{5}$ con el ánimo de presentar los proyectos

5 Se refiere a la participación de egresados, estudiantes y docentes de la línea en calidad de ponentes en: XII Encuentro de Geógrafos
Nubia Moreno Lache, Alexander Cely Rodríguez,

Liliana Rodríguez Pizzinato

en sus diferentes fases; encuentros de socialización con un carácter auto y coevaluativo en relación con el avance mismo de la indagación y con la formación como investigadores en los estudios sociales.

Otras actividades fundamentales que contribuyen en el proceso formativo de los estudiantes, radica en la realización de pasantías de investigación, entendidas como un escenario de profundización e interlocución con otras comunidades académicas interesadas en temas socio espaciales. Esta esfera que implica un esfuerzo, tanto del grupo a cargo de la línea como de los cursantes, constituye una oportunidad valiosa que influye en los resultados de la investigación y en la formación de los estudiantes, en su condición de investigadores.

Las pasantías realizadas han permitido la interacción de los estudiantes con otras comunidades académicas, así como la ampliación de horizontes de investigación en los campos socio-espaciales. En las comunidades internacionales se destaca el trabajo adelantado con la Universidad de Sao Paulo y de Goiania (Brasil), la Universidad Autónoma de México (UNAM), la Universidad Autónoma Metropolitana de México (UAM), el Instituto IPECAL (México), la Universidad Academia de Humanismo Cristiano (Chile). En el escenario nacional se ha adelantado esta modalidad de trabajo con la Universidad de Manizales (Colombia), la Universidad Nacional de Colombia y la Universidad Pedagógica Nacional (Bogotá, Colombia). Motivar e impulsar este trabajo en los estudiantes posibilita no solo intercambios conceptuales y metodológicos,

de América Latina celebrado en la ciudad de Montevideo, Uruguay (abril de 2009); en el I Coloquio Internacional de la REDLADGEO llevado a cabo en la Universidad de Sao Paulo, Brasil (junio de 2010); en la I Convención Nacional de Educación Geográfica y en el III Encuentro de Experiencias Significativas en la Enseñanza de las Ciencias Sociales y la Geografía realizado en la Universidad de Antioquia en Medellín (septiembre de 2010), en el XIX Congreso Colombiano de la Geografía realizado en la Universidad del Valle, Cali, Colombia (agosto de 2011); en el II Coloquio de la REDLADGEO realizado en la Universidad Academia de Humanismo Cristiano, en Chile (abril de 2012); en la II Convención Nacional de Educación Geográfica celebrado en la Universidad Pedagógica y Tecnológica de Colombia (septiembre de 2012); en el XIV Encuentro de Geógrafos de América Latina celebrado en la ciudad de Lima, Perú (abril de 2013), y en el XX Congreso Colombiano de la Geografía a realizarse en el mes de agosto del año en curso en la Universidad del Tolima, Ibagué, Colombia, (agosto de 2013). 
sino que también genera una ganancia cultural y de apertura a la producción, deliberación e impulso del trabajo epistémico a través de las redes académicas en diversos contextos; situación que indudablemente fortalece la formación de los investigadores.

Hace parte de la formación investigativa las asesorías y direcciones de tesis, las cuales se inician en el segundo semestre bajo un seguimiento riguroso que permite hacer los ajustes, evaluar los progresos y orientar la culminación de los proyectos, y a su vez, conforman un escenario de aprendizaje para el estudiante y el director que a la par nutren el panorama de investigación de Geopaideia a la luz de la preocupación por la construcción social del espacio.

Una lectura de las investigaciones adelantadas hasta la fecha muestra las siguientes tendencias (tabla 1). El eje identificado como contexto escolar, da cuenta de aspectos relacionados con las concepciones, imágenes, imaginarios y/o prácticas pedagógicas presentes en escenarios educativos, que reflexionan sobre conceptos espaciales y algunas experiencias escolares. En relación con este eje aparece el segundo, que está centrado en las concepciones y prácticas docentes. Indagar en esta perspectiva posibilita comprender formas y posiciones epistemológicas de la enseñanza de la geografía en Colombia, las que ameritan transformaciones tanto en sus concepciones como en su aplicación en la escuela.

El espacio público y sus esferas muestran relaciones y tensiones a través de acciones y apropiaciones de los ciudadanos; manifestaciones que son complejas, mutables y propicias para el estudio del espacio en tanto construcción social. Sectores urbanos como Santa Inés, Las Cruces, San Andresito, El Tintal y algunos de los parques metropolitanos en Bogotá reflejan particularidades y afinidades en el uso, dinámicas, y percepciones de sus habitantes, objeto tanto de lectura científica como de generación de comprensiones sobre las lógicas espaciales que pueden incidir, por ejemplo en la planeación urbana local y regional.

El eje relacionado con los sentidos de lugar, sugiere formas de construcción subjetiva de los espacios por parte de sus usuarios. Así, la memoria presente en los habitantes refleja los procesos de apropiación alrededor del surgimiento y consolidación del barrio en la ciudad, correspondientes a la condición de habitar no solo por ocupar un sitio, sino ante todo por construir, significar y plasmar en él huellas socio-territoriales. En la misma dirección, habitar la escuela a través de la imagen gráfica, supone diversas maneras de reconocimiento y simbolización que hacen parte de la producción subjetiva del espacio.

Una línea de discusión, que cobra fuerza en los ámbitos académicos en las últimas décadas, es la de los estudios de territorio, que en el caso particular de los hallazgos de investigación en la línea muestran preocupación sobre la territorialización, por ejemplo turística en sectores específicos de la ciudad (La Candelaria, Bogotá) y la apropiación material e inmaterial del territorio por parte de comunidades indígenas y LGBTI, que a su vez aportan otras visiones sobre los lugares y las formas de estar y ser ellos y que no están de manera frecuente presentes en los estudios tradicionales del espacio, por ende, son una ventana provocativa y provocadora para comprender al territorio.

La inmersión de la geografía en el ciber espacio indudablemente ha desplegado un campo necesario e interesante en la construcción social del espacio. Nuevas escalas, distancias, conceptos y usos emergen desde la virtualidad para la comprensión del espacio, y en ese campo las preocupaciones sobre cómo las personas comprenden el espacio real y vivido con el posibilitado en la web, propicia un escenario de deliberación fundamental que también ha cobrado presencia en la línea.

A pesar de que un número significativo de indagaciones se han realizado en la ciudad, es fundamental destacar las preocupaciones por los territorios rurales; búsquedas que pretenden comprender procesos y significaciones de lo rural en el contexto colombiano tomando como estudio de caso, por ejemplo, la situación de la tenencia de la tierra en el departamento del Tolima. De otra parte, la ruralidad y lo urbano forjan una relación dialógica a partir del 
espacio como construcción social al investigar sobre estos en la perspectiva del desarrollo turístico y del potencial que éste genera, pero también analizando las variables de afectación que produce.

Se destacan también en la línea perspectivas de indagación como geografía y literatura, así como la geografía de las religiones para la comprensión geográfica en cuanto construcción social. Temáticas de indagación que, evidentemente, demuestran tanto la dinámica del espacio como sus alternativas diver- sas de estudio; las que en campos de las geografías de la vida cotidiana revelan múltiples formas de la espacialidad.

Estas preocupaciones complejizan la investigación sobre el espacio geográfico, pero también nutren y dinamizan las concepciones y lecturas de la espacialidad. En el siguiente cuadro se reflejan los trabajos adelantados y en curso de la línea. (Tabla 1)

En cuanto a la dimensión rural del espacio y los estudios del territorio, se destaca la mirada sobre el

Tabla 1. Tendencias de investigación.

\begin{tabular}{|c|c|}
\hline $\begin{array}{l}\text { Estudiantes de la maestria } \\
\text { Línea "Construcción social del espacio" }\end{array}$ & Tendencias \\
\hline Lucia Mercedes Cáceres & \multirow{5}{*}{ Contexto escolar } \\
\hline Jorge Enrique Moreno & \\
\hline Luis Alfredo Bernal León & \\
\hline Marcela Cuevas & \\
\hline Eliana Catherine Álvarez Polo & \\
\hline Oscar Lombana Martínez & Prácticas y concepciones espaciales en los docentes \\
\hline César Ignacio Báez Quintero & \multirow{7}{*}{$\begin{array}{l}\text { Espacio público: concepciones, usos, } \\
\text { apropiaciones, significaciones }\end{array}$} \\
\hline Diego Ariza Coy & \\
\hline Felipe Castellanos Sepúlveda & \\
\hline Armando Galindo Joya & \\
\hline Carolina Moreno Cruz & \\
\hline Ruby Elena Varón Galvis & \\
\hline Gildardo Cortés & \\
\hline Andrea Montoya Carvajal & \multirow{2}{*}{ Sentidos de lugar } \\
\hline Deysi Paola Salazar Ariza & \\
\hline Edgar Salatiel Bejarano & \multirow{5}{*}{ Territorios y territorialidades } \\
\hline Yeimmy Andrea Pachón & \\
\hline Alvelayis Nieto Mejía & \\
\hline Tatiana Rodríguez Martín & \\
\hline Carlos Julián Parra M. & \\
\hline José Alejandro Vargas & Espacios y espacialidades en la web \\
\hline Jazmín Duarte & $\begin{array}{l}\text { Tenencia de la tierra y construcción } \\
\text { del espacio en contextos rurales }\end{array}$ \\
\hline Efraín Núñez Arévalo & Espacio geográfico y literatura \\
\hline Fabiola Córdoba Córdoba & Geografía de las religiones \\
\hline
\end{tabular}


campo como expresión del espacio rural, la cual lo ha considerado durante mucho tiempo como sinónimo de espacio agrario y aun a finales del siglo XX no se puede negar que sea cierto en muchos casos, ya que el uso del suelo dedicado al desarrollo de las actividades agrarias y forestales ha mantenido un primer lugar en el PIB de la mayoría de los países del mundo. Sin embargo, la

Producción agrícola progresivamente se ha especializado y presenta dos modelos claramente diferenciados. En los países desarrollados, donde la densidad de población urbana es muy elevada, el espacio rural muestra una amplia variedad de usos, entre los que la agricultura no es la única actividad económica. (García, Tulla, Puyol y Valdovinos, 1995, p. 27)

En tal sentido, el espacio rural hoy no se puede considerar como una entidad totalmente independiente, sino que es un complemento del espacio urbano especialmente en las sociedades industrializadas. Por ello, las diferencias entre ambos son cada vez menos visibles. A la descentralización de la vivienda se añade la de las actividades productivas, como la industria y algunos servicios, en especial relacionados con el ocio que cobran un papel protagónico en los espacios rurales.

Sin embargo, el modelo en los países no industrializados es un poco distinto; el espacio sigue considerándose de manera dual, ya que aún se observa una mayor diferencia entre la organización de las actividades económicas de intercambio en el marco del mercado internacional y las que se orientan al consumo local. Tanto en las ciudades como en el campo, se delimitan los espacios de acuerdo con su orientación.

En la medida que se han transformado las relaciones ciudad-campo, los usos de suelo se han modificado en las áreas rurales guardando las proporciones entre países industrializados y no industrializados. Lo anterior, explicita las relaciones de simbiosis que se manifiestan entre el espacio urbano y rural.

Estos aspectos juegan un papel importante en los proyectos de investigación que reflexionan alrede- dor de esta dimensión espacial, tanto en el contexto escolar como en la organización comunitaria y campesina, demostrando el interés por problematizar la espacialidad en este ámbito y transformar las concepciones y acciones que impactan progresivamente distintos contextos.

En cuanto a la categoría de territorio, se parte de su acepción latina, que al principio designaba la zona que rodeaba a una ciudad y estaba bajo su jurisdicción y se aplicaba a las ciudades-estado del mundo clásico. En su acepción moderna ya no se asocia con el entorno de la ciudad, ya que supone una división del poder político: "el territorio es la tierra que pertenece al que gobierna un Estado, significado que se remonta a 1494 aproximadamente al momento en que nace la economía-mundo" (Taylor, 1993, p. 132).

La unión de territorio y soberanía surge en el siglo XVI y se consolida con el Tratado de Wesfalia (1648), que reconocía que cada Estado era soberano en su territorio, es decir, que la interferencia en los asuntos interiores era considerada el primer delito contra el derecho internacional. En consecuencia, se reconoció a Europa parcelada en cerca de trescientas unidades soberanas. Esta fue inicialmente la base territorial del sistema interestatal moderno: el primer mapa político mundial. El territorio tiene, desde esta perspectiva, dos funciones: la seguridad que permite circular los recursos con estabilidad y de un modo más completo, y permitir el nacimiento del mercado mundial.

Otra manera análoga de considerar el territorio es relacionarlo con una porción de espacio geográfico, sobre el cual se ejerce o se pretende ejercer el control político. Este control, relativo y jerarquizado, puede ser pretendido por una persona, un partido político, un grupo social o un Estado.

Como consecuencia del ejercicio de la soberanía se configura la territorialidad, como el grado de control de una determinada porción de espacio geográfico por una persona, un partido político, un grupo social o un Estado, la cual está condicionada por las relaciones de poder político, de apropiación y de expresión espacial. Cabe señalar que el territorio y la territorialidad también pueden ser producidos 
socialmente, permeando las estructuras institucionales mencionadas. Esto significa que los seres humanos pueden construir multiplicidad de territorios de acuerdo con su accionar en ellos, con los signos y símbolos que se tejen en él, con las narrativas que circulan y de cierta manera se posicionan en el lugar, con la memoria acumulada que refleja la experiencia colectiva de la sociedad.

Por esta razón, las territorialidades que se forman no coinciden con límites político- administrativos determinados por las instancias estatales, sino con los imaginarios colectivos que trazan una marca simbólica $\mathrm{y}$ definen territorios cognitivos especiales y significativos para sus actores.

En tal sentido, los proyectos de investigación que se han preguntado por el territorio y la territorialidad están descubriendo nuevas formas de organización desde la subjetividad, desde las configuraciones territoriales que dialogan o se ponen en tensión con las prácticas institucionales y que empiezan a mirar lo espacial como una producción de territorialidades de las voces escondidas en la sociedad, como las minorías, los grupos barriales o las periferias urbanas o rurales.
Nubia Moreno Lache, Alexander Cely Rodríguez, Liliana Rodríguez Pizzinato

En conclusión, las tendencias identificadas son ricas tanto en su naturaleza como en sus referentes conceptuales y metodológicos, reafirmando la complejidad de la categoría espacial y la necesidad de ahondar en sus perspectivas al igual que en su lectura y comprensión. Un número significativo de esos trabajos culminaron, aportando elementos valiosos de reflexión, generando apertura a estudios que amplíen los horizontes trazados, a la proyección de estudios comparativos y al enriquecimiento de la concepción del espacio como resultado de la interacción humana, es decir, como constructo social.

Las investigaciones culminadas (Tabla 2) y las que están en curso permiten visualizar posibilidades y realidades espaciales interesantes, en tanto ahondan en la lectura de la ciudad y de sus territorios a diversas escalas, percepciones y expresiones. Variables que no necesariamente se pueden contemplar en una lectura tradicional del espacio geográfico y que poseen un enorme potencial en la indagación de los territorios. Situación que se evidencia a continuación:

Tabla 2. Investigaciones finalizadas LCSE ${ }^{6}$.

\begin{tabular}{|c|c|}
\hline Investigación & Espacio social y lúdica: Lectura de un escenario público de Bogotá \\
\hline Autor & Cesar Ignacio Baez Quintero \\
\hline Propósito & $\begin{array}{l}\text { El objetivo del trabajo es comprender cómo, por medio de la lúdica, los habitantes de Bogotá identifican, } \\
\text { apropian y valoran el parque metropolitano Simón Bolívar. }\end{array}$ \\
\hline Metodología & $\begin{array}{l}\text { La investigación realizo un trabajo etnográfico para la recopilación de la información, por medio de diarios de } \\
\text { campo, entrevistas (sistematizadas por medio del Atlas ti), fotografías y superposición cartográfica. }\end{array}$ \\
\hline $\begin{array}{l}\text { Algunos } \\
\text { resultados }\end{array}$ & $\begin{array}{l}\text { Los resultados de la investigación se organizan en tres niveles: } \\
\text { - Aportes teóricos que el trabajo desarrolló desde la lúdica y el espacio social } \\
\text { - Aportes derivados de la investigación, los cuales pretenden ser una contribución a las políticas y planes de } \\
\text { organización del parque } \\
\text { - Aportes en relación con el proceso de formación como investigador y la propuesta de la Maestría en Estu- } \\
\text { dios Sociales. }\end{array}$ \\
\hline Investigación & La geografía en los textos escolares de ciencias sociales (1995-2008), tendencias y posibilidades \\
\hline Autor & Lucía Mercedes Cáceres Copete \\
\hline
\end{tabular}

6 Los informes finales del proceso de investigación adelantado por cada uno de los estudiantes (hoy egresados) pueden ser consultados en la biblioteca de la Universidad Pedagógica Nacional, toda vez que una copia de estos trabajos debe ser entregado en dicha instancia como parte de políticas de socialización del conocimiento que se produce en el programa de la Maestría y como política institucional de los programas de formación. 
Universidad Pedagógica Nacional

Facultad de Humanidades

\section{Propósito \\ Metodología}

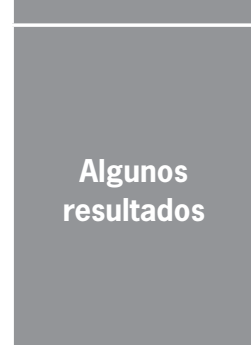

Investigación

Autor

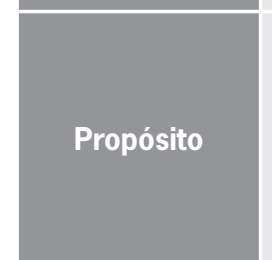

Metodología

Algunos

resultados

Investigación

Autor

Propósito

Metodología

Metodología

Algunos

resultados

Investigación

Autor
El trabajo de investigación tiene como objeto de estudio a los textos escolares de ciencias sociales y las formas como aborda la geografía por medio de categorías que son construidas en la disciplina. El objetivo general es interpretar las características del conocimiento geográfico escolar presente en los textos de ciencias sociales en grados quinto, sexto y séptimo de educación básica en la ciudad de Bogotá, entre 1995 y 2008.

El trabajo se fundamenta en el paradigma hermenéutico, caracterizado por enfocarse en describir, analizar e interpretar hechos sociales producidos por sujetos en contextos particulares.

Desde la parte investigativa, la tendencia que más tiene presencia dentro de los contenidos geográficos es de tradición regionalista, la cual se hace explicita a través de su objeto de estudio, la región, que predomina como unidad de análisis de las relaciones entre la naturaleza y las sociedades. Además, se comprueba que existe un fuerte arraigo en la geografía física, ya que temas como generalidades de la tierra o del país en cuanto a aspectos como relieve, hidrografía y clima, continúan teniendo vigencia dentro de los procesos de enseñanza de la geografía. En la reflexión sobre la investigación y su objeto de estudio, se asume el texto escolar como herramienta que permite hacer acercamientos a contenidos geográficos que tienen legitimidad en la escuela y en las representaciones e imaginarios de estudiantes, padres de familia y profesores.

La ciudad: Cotidianidad, experiencias y percepciones de la niñez

Jorge Enrique Moreno Rojas

La investigación está planteada desde las inquietudes que el investigador recoge de su experiencia en la enseñanza de la geografía en la escuela y de los resultados investigativos en cuanto a la didáctica de la geografía en el desarrollo del pensamiento espacial a partir de la construcción de conceptos geográficos. Por ello, el problema de investigación se dirige a identificar la incidencia de las vivencias y percepciones espaciales urbanas cotidianas de los estudiantes entre los 10 y 12 años de edad, del colegio Fundación Colombia ubicado en la ciudad de Bogotá D.C., en el proceso de construcción de sus conceptos de ciudad.

Esta pesquisa se inscribe dentro del ámbito de los estudios cualitativos con carácter interpretativo. Utiliza la encuesta, que permite realizar un primer acercamiento al conocimiento de las vivencias de los estudiantes y una aproximación a las imágenes, que aunque verbalizadas, son fuente importante para el rastreo del proceso de construcción de las mismas por parte de los estudiantes.

Los resultados obtenidos permiten reconocer la presencia de una "ciudad de ellos"; una referencia a la marcada confluencia de aquello que existe en la cotidianidad de niños y niñas: emociones, vivencias, sentimientos, experiencias de ciudad. Es así como la percepción que tienen los niños y las niñas de su ciudad determina que esta tenga un carácter definido y particular.

Territorialización del turismo en el Centro Histórico de Bogotá

Edgar Salatiel Bejarano Martínez

La investigación busca comprender las complejas relaciones que se dan entre turismo y territorio al interior de la zona del Centro Histórico de Bogotá destinada para usos turísticos. Estas relaciones van desde la transformación física, debido a la implementación de infraestructuras y servicios, hasta la transformación de aspectos culturales, sociales y ambientales, como resultado de las actividades de los turistas y visitantes.

Este trabajo se inscribe en el segundo grupo, el de las ciencias histórico-hermenéuticas. El ejercicio está orientado por un interés práctico, ya que se busca interpretar el sentido de una realidad dada para los sujetos implicados en ella, con la intención de obtener nuevos conocimientos que puedan servir de guía y orientación para la acción.

Se desarrollan algunos aportes relacionados con la recuperación de la habitabilidad del centro histórico, a partir del desarrollo de un turismo sostenible y bien planificado; el mejoramiento de la cultura turística tanto de habitantes como de visitantes, y la relativización de la importancia del crecimiento económico como único objetivo de la planificación del desarrollo turístico en la localidad.

Un lugar vivido, un lugar construido

Deysi Paola Salazar Ariza 


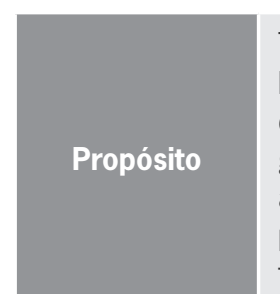

Teniendo en cuenta la pregunta que orienta esta investigación: ¿Cómo ha sido el proceso de construcción de lugar que ha tenido la población del barrio Lucero Bajo en Bogotá, a partir de las dinámicas socio-históricas que se han consolidado en las experiencias de sus habitantes?, se desarrolló el proyecto a partir de los siguientes ejes temáticos:

a) Entre las montañas. b) Mil Colores al sur de Bogotá. b) Y nació un lucero. c) La lucha por los servicios públicos. d) La vivienda, porque la autoconstrucción está presente. e) Construyendo lugar y sentidos de lugar. f) Filias y fobias en el barrio Lucero Bajo. g) Usos de suelo en el barrio Lucero Bajo.

Para el trabajo de investigación se aborda una metodología cualitativa, a partir de la cual se determinaron las categorías de análisis para llevar a cabo la lectura de información y la elaboración de resultados, permitiendo obtener las claves para interpretar las formas en que los seres humanos construyen lugar, a partir del estudio de caso que se propuso. Dicha lectura se realizó por medio del uso del software Atlas ti, el cual permitió la elaboración de ocho códigos de análisis que surgieron de la pregunta de investigación unida a los tres obje-

Metodología tivos específicos y preguntas de la entrevista. Estos códigos son: orígenes del barrio, construcción de lugar, referentes de lugar, experiencias, significados, filias, fobias, cotidianidad, viviendas, los cuales se tomaron como referentes para clasificar la información dentro de tres grandes categorías de análisis: lugar, sentidos de lugar, filias y fobias. Por otra parte, en la propuesta metodológica se realizó un estudio de caso y se implementaron técnicas de investigación como entrevista no estructurada, entrevista a profundidad, observación directa, trabajo sobre planos y análisis de archivo fotográfico.

- La casa, la cuadra, el barrio, la ciudad, se han convertido para los seres humanos en lugares; en la medida que adquieren un significado y son parte de sus vivencias diarias.

- Se rescató parte de la memoria de una comunidad que hizo de una porción del espacio, un centro de actividades, relaciones sociales, experiencias individuales y compartidas.

Algunos resultados

- Las experiencias en un marco de la cotidianidad son las que permiten la construcción de lugar, generando dinámicas de apropiación, reconocimiento, apego, desapego, amor y rechazo por una porción de este gran espacio.

- La relación entre el ser y el espacio desde el punto de vista de la geografía humanística, es una experiencia mediada por las vivencias, aquellas que permiten hacer del espacio una construcción social, que repercute y da como resultado la construcción de lugar.

Investigación

Prácticas sociales y ciudadanía en espacios públicos de la ciudad de Bogotá, D.C.

Autor Diego Ariza Coy

Trabajo de investigación centrado en el análisis e interpretación de prácticas sociales en los espacios públicos

Propósito de El Tintal, zona emplazada en el occidente de la ciudad de Bogotá, con el fin de establecer su construcción como lugar del habitar y como escenario para el ejercicio de la ciudadanía.

Se enmarca dentro de un horizonte epistemológico de corte cualitativo-interpretativo asociado con la sociología urbana, y que se enfoca desde el método etnográfico para interpretar formas de apropiación sobre el espacio público relacionadas con la construcción de lugares para el habitar en escenarios para la socialización y la ciudadanía. Se implementó la observación participante y no participante junto con la aplicación de registros de campo, entrevistas cualitativas enfocadas y encuestas estructuradas semi abiertas que contribuyeron a la construcción de cartografía digitalizada que muestra los principales hallazgos de la investigación.

Algunos

resultados

El Tintal es un espacio público que se construye socialmente a partir de la experiencia espacial de sus habitantes. El Tintal como un espacio público vivido (Ramírez, 2006) lleva implícita la idea de hablar de una construcción social de la ciudad donde se reflejan elementos de la subjetividad asociados con percepciones hacia lo que implica el habitar desde el quehacer de la vida cotidiana.

Investigación

Ejercicios de poder en el espacio público: Experiencias desde el trabajo informal en San Andresito de la carrera 38 en Bogotá, D.C.

Autor Carolina Moreno Cruz

La investigación busca comprender la configuración del espacio público a partir del ejercicio de poder y su construcción por parte del trabajador informal, caracterizando las relaciones de poder, los usos que hacen del espacio público y las construcciones históricas que se dan en un lugar determinado, que en este caso es San Andresito de la carrera 38 en Bogotá. 
Universidad Pedagógica Nacional

Facultad de Humanidades

Metodología

Algunos

resultados

Investigación

Autor
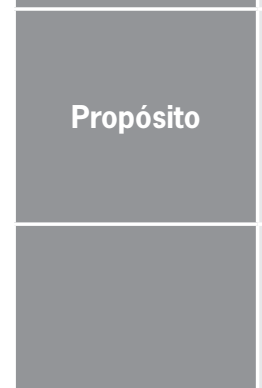

Metodología

resultados

Investigación

Autor

Propósito

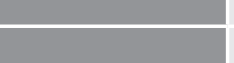

Metodología
El trabajo está guiado por un enfoque cualitativo de corte interpretativo, y el método de investigación es la etnografía.

Esta investigación enmarcada en una lectura crítica e interpretativa del espacio público generó nuevas herramientas para analizar las cuestiones urbanas, haciendo énfasis en la relación geografía y acción política, pues cada uno de estos elementos fortalece los estudios sociales y dinamiza las entradas a la lectura de lo cotidiano, que visto bajo el lente investigativo da como resultado hallazgos que pueden de una u otra forma generar proyectos a largo plazo con las comunidades.

Desrepres -arte con trazos y manchas. Habitar la escuela por medio de la imagen gráfica

Andrea Montoya Carvajal

El objetivo de esta investigación se orienta a interpretar la forma de habitar los estudiantes la escuela por medio de la imagen gráfica, para lo cual es necesario: describir los significados que construyen los autores de la imagen gráfica en la escuela antes de la emergencia de esta, describir los significados que construyen los no autores de la imagen gráfica posterior a la emergencia de esta y establecer los significados semejantes y diferentes que se construyen antes y después de la imagen gráfica.

Se realiza una investigación de tipo cualitativa con un enfoque interpretativo-etnográfico, en esta se aborda el estudio del espacio desde el enfoque de la geografía humanística, para describir cómo habitan los estudiantes la escuela por medio de la imagen gráfica. Para hacer visibles los significados que se generan antes y después de la imagen gráfica, se diseña una ruta metodológica en la que se plantean tres fases de investigación en coherencia con los objetivos específicos: Primero se desarrolla una práctica investigativa que posibilita recoger información de la cotidianidad de la escuela, para lo cual se acude inicialmente a la observación para identificar las huella en los diferentes lugares del contexto escolar determinando los modos de hacer y estableciendo una tipología de la imagen gráfica. En una segunda fase, al identificar las huellas gráficas, se delimita la población en la que predominan las manifestaciones por medio de este lenguaje, la cual corresponde a los grados de quinto a undécimo del Colegio de los Andes (Fontibón), por lo cual, se acude a la encuesta para categorizar a los sujetos en autores o no autores de la imagen gráfica. En la tercera fase, una vez reconocidos los sujetos, se organizan grupos focales para identificar los significados que tanto autores como no autores asignan a la imagen gráfica para finalmente implementar un ejercicio cartográfico (mapeo) con la intención de identificar significados del lugar de manera colectiva.

Se encuentra, entre otros, que en el ámbito escolar el uso de la imagen gráfica se hace evidente en los diversos sitios por los que transitan los estudiantes en la cotidianidad, dando cuenta de una forma de habitar la escuela, cuando dichos sitios se transforman en lugares, al asignarles significados a través de la imagen gráfica. En relación a ello, dicha imagen se convierte en lo que en términos de Lindón (2006) es la huella de un holograma espacial, el cual permite estudiar la construcción social de los lugares. De igual manera, desde la experiencia del sujeto, se encuentra que el holograma espacial emerge de la huella gráfica y se configura por unos elementos que se presentan como las partes que contienen información sobre el objeto holográfico, es decir, sobre la huella gráfica.

Las Cruces: Un territorio de estudio para la comprensión de la espacialidad

Ruby Elena Varón

La investigación busca comprender la construcción social de la espacialidad cotidiana del barrio Las Cruces, desde representaciones espaciales y prácticas cotidianas de sus habitantes, en un territorio que es transformado por la política de renovación urbana.

La investigación maneja un enfoque cualitativo-interpretativo al articular diferentes elementos de tipo geográfico y aspectos sociológicos en relación con el sujeto que expresa y representa las prácticas cotidianas producto de las experiencias espaciales en el barrio Las Cruces. Se aborda una dimensión simbólica que permite indagar por la vida cotidiana, expresada mediante retóricas, discursos, lógicas y narrativas desde los cuales los individuos actúan e interpretan al otro y al mundo. En consecuencia, se destaca el empleo de la observación participante, la entrevista biográfica, los esbozos de mapas y la utilización de los programas computacionales ArcMap, ArcGis, y Atlas ti para comprender la construcción social de la espacialidad cotidiana del barrio Las Cruces, desde representaciones espaciales y prácticas cotidianas de sus habitantes, en un territorio que es transformado por la política de renovación urbana. 


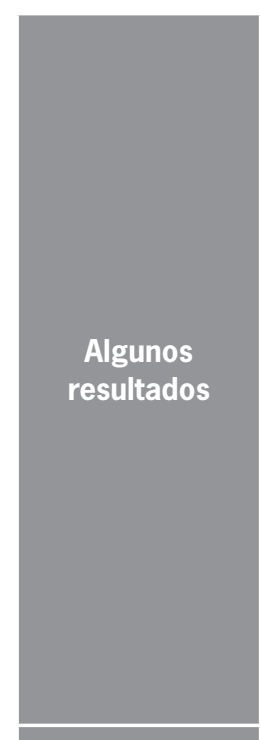

Investigación

Autor

\begin{tabular}{|c|}
\hline Autor \\
\hline Propósito \\
\hline
\end{tabular}

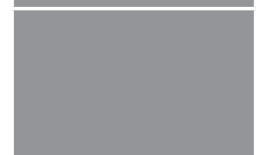

Metodología

resultados

Investigación

Autor
En cuanto al componente investigativo:

1. El sujeto y el espacio están vinculados en relaciones complejas que los complementan mutuamente.

2. Los hallazgos muestran que es posible estudiar los espacios a través de las prácticas cotidianas de los sujetos y sus representaciones espaciales, debido que permiten recorrer significados, memorias y experiencias espaciales que demarcan geográficamente la construcción social del espacio como resultado de intereses, relaciones y prácticas.

3. El barrio Las Cruces hoy sufre un proceso de deterioro, producto de las marcadas problemáticas sociales e históricas, pero a su vez consecuencia del desplazamiento de otras problemáticas de barrios aledaños intervenidos por proyectos de renovación urbana.

En los roles de investigadora y docente:

1. En el contexto escolar o comunitario los elementos teóricos y metodológicos propuestos son una fuente para indagar sobre las realidades barriales y proponer compresiones y reflexiones que lleven al sujeto a participar en sus comunidades de manera activa para la planeación de los espacios y el reconocimiento de la ciudad.

Aportes para la maestría y la línea construcción social del espacio:

El sujeto es parte central para la compresión del espacio y la construcción de la realidad cotidiana. Desde una visión subjetiva cuestiona a los estudios sociales y su relación con la geografía, el urbanismo, la gestión y la planeación urbana.

Las representaciones sociales en la configuración de las prácticas pedagógicas de los docentes de ciencias sociales: Caso de cuatro instituciones educativas de los municipios de Arauca y Arauquita (Colombia)

\section{Oscar Iván Lombana Martínez}

Reconocer las representaciones sociales que configuran prácticas pedagógicas en la enseñanza de la geografía de los docentes de ciencias sociales en cuatro instituciones educativas de los municipios de Arauca y Arauquita.

Es un ejercicio de investigación que se ubica en el paradigma cualitativo-interpretativo de enfoque biográfico-narrativo que tiene como objetivo general reconocer las representaciones sociales que orientan las prácticas pedagógicas de los docentes de ciencias sociales en los municipios de Arauca y Arauquita. Esta estrategia metodológica contempla tres tipos de aproximaciones con los sujetos del estudio: las historias de vida, observación de clase, y un ejercicio documental en torno a las instituciones educativas y su desenvolvimiento cotidiano. El trabajo se desarrolla con base en las historias de vida de cuatro profesores de ciencias sociales cuyos relatos permiten establecer cuáles son las representaciones que orientan sus prácticas, partiendo de la idea de que estas prácticas son intuitivas, categoría que atraviesa el ejercicio y que permite pensar que las teorías pedagógicas y la formación inicial docente no se constituyen necesariamente en rutas que impacten las practicas de los maestros en su quehacer cotidiano.

- La formación de maestros de ciencias sociales debería contemplar una formación y cualificación permanente de sus prácticas pedagógicas para responder a las transformaciones del mundo actual.

- En este sentido, la formación de los maestros debería caracterizarse por contener un alto componente investigativo propendiendo por la popularización de las comunidades de la construcción del conocimiento.

- Por ser un aprendizaje permanente debería contemplarse desde la formación docente inicial y atravesar todos los estadios de desarrollo profesional del maestro, incluso su vinculación laboral, y a lo largo de su vida.

- En este sentido, dicha formación debería propender por una actualización disciplinar y pedagógica constante, al mismo tiempo que ofrecer escenarios de reflexión didáctica, en la que la observación solidaria, es decir, en la que la participación de sus compañeros y las comunidades educativas en general sea un hábito, además de un componente fuerte de asistencia técnica (en diferentes ámbitos).

- La formación inicial debería contener escenarios de práctica temprana, en la que los mismos formadores de formadores trabajen con base en los presupuestos metodológicos que se espera se apliquen en la escuela; los maestros universitarios, en su mayoría, no reconocen o no aplican las metodologías que esperan que sus estudiantes apliquen en su labor como educadores.

Territorio transgénero: Un territorio vivo en el barrio Santa Fe

Jeymmy Andrea Pachón Torres 
Universidad Pedagógica Nacional

Comprender cómo es construido el territorio del barrio Santa Fe (carrera 22 entre calle 14 y 16 a) por parte de la población transgenerista joven y adulta de la Corporación Opción.

La investigación asume un enfoque cualitativo-interpretativo que ve a la realidad social como "un proceso constantemente reconstruido, un flujo continuo de experiencia y acción" (p. 45). Se deduce que es un estudio de corte etnográfico que se apoya en las técnicas de la entrevista biográfica y la "observación sistemática". La entrevista biográfica por su lado pretende "recopilar, analizar y establecer las intersubjetividades y apuestas de vida de los sujetos dentro del territorio" (p. 46); enfatiza en la narración, el lenguaje, la estética y la relación cercana entre entrevistado y entrevistador. Mientras que la observación sistemática se plantea como una estrategia para definir y redefinir categorías en el proceso investigativo que no es precisamente una observación participante, pero sí un ejercicio de valorar el habitar, la estética urbana y los actores sociales en la cotidianidad con ayuda de la fotografía.

- En cuanto a la experiencia de las mujeres transgeneristas en el barrio Santa Fe se sostiene que el género y sus componentes de identidad no son solo un proceso de configuración de la subjetividad sino de las dinámicas de negociación y conflicto que encierra el espacio social.

- La territorialidad de las mujeres transgeneristas está atravesada por la noche ilegal y subterránea y el día legal, es decir, por los ritmos de las relaciones sociales que a diario dan uso al territorio del barrio Santa Fe; la prostitución, el turismo sexual y el micro tráfico de drogas generan redes de normas y acuerdos entre los actores sociales.

Algunos

resultados

- Las políticas públicas de atención a las mujeres transgeneristas no dan cuenta de la cotidianidad de los actores sociales y se enfocan en el tratamiento del riesgo; esto profundiza las dinámicas de violación de derechos humanos. La lectura de las territorialidades en juego es clave para lograr avances en la atención a esta población.

Fuente: RAES Trabajos de investigación-Egresados LCSE

Se puede afirmar que los senderos transitados a partir de las investigaciones adelantadas se han convertido para el grupo Geopaideia, así como para los cursantes adscritos a la línea, en un interesante escenario que progresiva y paulatinamente devela formas múltiples de lo espacial. Develaciones que implican grandes compromisos en la medida que el asunto del espacio involucra un reposicionamiento tanto de sus concepciones como de su aplicación en la vida y en las vivencias cotidianas de las personas. Sería posible pensar que éstas insinúan alternativas para el reconocimiento, la interpretación y comprensión de la espacialidad como también para aportar en una educación espacial, no limitada a la escuela como institución únicamente encargada de ello, sino extendida a los habitantes de los lugares:

La educación podría constituirse en una ventana hacia la toma de conciencia de las experiencias espaciales cotidianas. Una educación geográfica de esta naturaleza podría ser parte de los procesos de socialización de los individuos en sentido amplio: así como se aprende el lenguaje, a reconocer los códigos y marcos sociales y a comunicarse con los otros, podríamos aspirar a una socialización espacial. (Lindón; 2009, p. 161)
La actual cohorte se encuentra en el proceso de formulación de la investigación y sus respectivos ajustes, acompañado por el desarrollo de actividades como la consulta de información para la construcción del estado del arte, de los referentes teóricos y conceptuales y el diseño metodológico. A pesar de ello es posible identificar matices nuevos, otros que ahondan en los ya identificados o complementarios a la indagación del espacio en cuanto construcción social, como se anota a continuación: (Tabla 3 )

\section{Algunas consideraciones finales}

La experiencia que ha obtenido Geopaideia en la reflexión y estudio del espacio en tanto construcción social le permite afirmar que la espacialidad con las ventajas existentes, como también con las debilidades presentes en la comunidad académica, es consecuencia del desarrollo de paradigmas como el humanístico, pues logra instaurar y afincar preocupaciones espaciales desde la perspectiva de los sujetos. Indudablemente, esta condición ha motivado en los últimos años diversos estudios, mayores experiencias y, por ende, un cúmulo más amplio de 
Tabla 3. Investigaciones LCSE en curso.

\begin{tabular}{|l|l|}
\hline \multicolumn{1}{|c|}{ Autor } & \multicolumn{1}{c|}{ Intención investigativa } \\
\hline Luis Alfredo Bernal León & Una propuesta curricular para la enseñanza del espacio geográfico \\
\hline Marcela Cuevas & La enseñanza y aprendizaje de la geografía en el contexto rural \\
\hline Alvelayis Nieto Mejía & $\begin{array}{l}\text { Propuesta para el desarrollo integral del turismo sostenible en la provincia del norte } \\
\text { del Tolima como aporte social, económico, cultural y ambiental }\end{array}$ \\
\hline Gildardo Cortés González & $\begin{array}{l}\text { El portal de Usme como centralidad a partir de la cotidianidad de los habitantes de } \\
\text { barrio La Fiscala }\end{array}$ \\
\hline Tatiana Rodríguez Martín & Territorios y territorialidades en la plaza de Usaquén \\
\hline Efraín Núñez & $\begin{array}{l}\text { Concepciones y representaciones del espacio geográfico en la literatura. Caso Muni- } \\
\text { cipio de La Mesa, Cundinamarca }\end{array}$ \\
\hline Julián Parra & $\begin{array}{l}\text { Concibiendo el territorio del Centro Histórico de la localidad de Usaquén, desde la mi- } \\
\text { rada de sus habitantes transitorios: caso de los estudiantes del IED General Santander }\end{array}$ \\
\hline Eliana Catherine Álvarez Polo & Textos escolares y análisis del discurso desde el espacio afrocolombiano \\
\hline Fabiola Córdoba Córdoba & $\begin{array}{l}\text { Geografía de las religiones: espacios y espacialidades desde la religión en la ciudad } \\
\text { de Bogotá }\end{array}$ \\
\hline
\end{tabular}

información para continuar pensando la relación de diálogo entre los seres humanos y los espacios que habitan.

"Precisamente porque las geografías son simbólicas, es decir, humanas, éstas no sólo definen sino que describen las distintas maneras de ser en el mundo" (Yory, 2007, p. 76); esta afirmación aportada por y para el estudio de la espacialidad posibilita reconocer en el ejercicio adelantado por la línea "Construcción social del espacio" que los tránsitos investigativos resultan no solo necesarios sino también novedosos, y que, justamente por ello es necesario ahondar, escudriñar, descubrir y avanzar en las indagaciones del espacio.

Se evidencia que los espacios tanto cercanos como lejanos son un potencial interesante de conocimiento que no necesariamente han sido develados en los panoramas académicos y menos aún en los de la cotidianidad. Las investigaciones reafirman que por lo general invisibilizamos los lugares cercanos, asumiendo que en ellos todo está conocido; por el contrario en estos espacios hay un amplio panorama por descubrir.

Panorama que se articula con la reflexión sobre el espacio como condición para el desarrollo de la vida cotidiana; en tal sentido, esta se constituye en pieza clave para la identificación de comportamientos y actitudes espaciales, que exigen cada vez más ser abordados desde la academia, y a su vez que ésta aporte elementos y categorías de comprensión para el diseño y ejecución de planes y propuestas de desarrollo local, municipal, regional e incluso nacional.

En cuanto a los logros y limitaciones en el plano metodológico, es interesante reafirmar que la investigación es un proceso amplio, nutrido, novedoso e impactante que no responde a cánones estáticos, sino que acompañado del rigor investigativo insinúa nuevas formas de acercarse a la realidad en sus múltiples facetas. Es por ello que las apuestas de indagación plasmadas en los programas de formación avanzada reposicionan la vigencia y necesidad de la Maestría y de investigaciones que impacten en los diferentes niveles espaciales en los que desarrollan la vida las personas. A su vez, se requiere que la Maestría y las investigaciones que genera sean un escenario propicio para la formación continua tanto de estudiantes como de docentes a cargo de los mismos. Aplicando la metáfora de Ortiz a la investigación damos cuenta, precisamente, de la trascendencia y relevancia del ejercicio investigativo:

Puedo tejer con pocos hilos: mi tela quedará entonces algo empobrecida, monocromática. Cuan- 
do junto colores y espesores diferentes, altero su granulado, su matiz. Una tela rica posee tonalidades y sombras, su superficie es irregular y rugosa. Los hilos, o mejor, su entrelazamiento, hacen el resultado final. Cuando escribimos, trabajamos con un conjunto de ovillos a nuestra disposición. Está claro que siempre existe el riesgo de perderse en la búsqueda de esas referencias textiles. Por eso se impone una selección juiciosa, se trabaja con un número limitado de ovillos. La escritura es el resultado de una costura, de la conjunción entre la aguja y los hilos. (Ortiz, 2004, p. 14)

La formación investigativa en la línea "Construcción social del espacio" incide también en concepciones sobre la enseñanza del espacio y en la educación geográfica, afirmando que ésta no es solo asunto de la escuela, si bien es su principal escenario, se demanda desbordarla y lograr que los habitantes comprendan cada vez más la complejidad del lugar en el que desarrollan sus vidas, que se apueste y aporte en la educación espacial de los habitantes de los lugares y territorios; como también es necesario impactar progresiva, acertada y ascendentemente en instituciones, organizaciones, políticas públicas y planes de desarrollo que incluyan el espacio y sus habitantes con el deseo de alcanzar verdaderas arquitecturas del espacio, ese es, posiblemente, el reto más interesante de un programa como el que se ha expuesto en la presente disertación.

\section{Referencias}

Abad, H. (2003). Angosta. Bogotá: Editorial Planeta.

Capel, H. y Urteaga, L. (1985). Las nuevas geografías. Barcelona: Salvat Editores.

Delgado, O. (2003). Debates sobre el espacio en la geografía contemporánea. Bogotá: Universidad Nacional de Colombia.

Estébanez, J. (1983). Tendencias y problemática actual de la geografía. Barcelona: Editorial Cincel.

Franco, M. (1997). Trayectoria de la geografía. En E. Rodríguez, et al., Geografía y ambiente. Enfoques $y$ perspectivas (pp. 11-30). Bogotá: Ediciones Universidad de la Sabana.
García, M., Tulla, I. Puyol, A. y Valdovinos, N. (1995). Geografía Rural [Serie Espacios y Sociedades n. ${ }^{\circ} 10$ ]. Madrid: Editorial Síntesis, S.A.

Lindón, A. (2006). La espacialidad de la vida cotidiana. Hologramas socio-territoriales de la cotidianidad urbana. En J. Nogue y J. Romero, Las otras geografías (pp. 425-445). Valencia: Tirant lo Blanch.

Lindón, A. (2009). La educación geográfica y la ampliación de las Terrae Cognitae personales. En M. Garrido, La espesura del lugar. Reflexiones sobre el espacio en el mundo educativo (pp. 153-169). Santiago de Chile: Universidad Academia de Humanismo Cristiano.

Ortiz, R. (2004). Taquigrafiando lo social. Argentina: Siglo XXI editores.

Taylor, P. (1993). Geografía política. Economía-mundo, Estado-nación y localidad. Madrid: Trama Editorial.

Yory, C. M. (2007). Topofilia o la dimensión poética del habitar. Bogotá: Editorial Pontifica Universidad Javeriana. 